\title{
Novel transdermal device for delivery of triamcinolone for nail psoriasis treatment
}

Yik Weng Yew ${ }^{* 1,2}{ }_{M B B S}$, Crystal Zhen Yu Phuan ${ }^{* 1}{ }_{M R C P}$, Xiahong $\underline{\text { Zhao }}{ }^{1}{ }_{\text {PhD }}$, Eugene Sern Ting $\underline{\operatorname{Tan}}^{1}{ }_{M B B S}$ (Singapore),

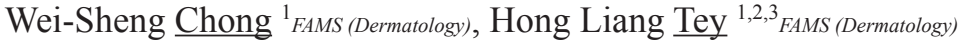

\begin{abstract}
Introduction: Nail psoriasis treatment is challenging due to difficult drug delivery and systemic therapy toxicities. Self-dissolvable microneedle patches embedded with corticosteroids offers a potentially rapid, minimally invasive drug delivery platform with good efficacy and minimal adverse side effects.

Methods: We conducted a 4-month prospective randomised controlled trial. Subjects with psoriatic nails were randomised to receive microneedle device delivered topical steroids on one hand and control treatment (topical Daivobet gel) on the other. Two independent dermatologists blinded to the treatment assignment scored their Nail Psoriasis Severity Index (NAPSI) during visits at baseline, 2 and 4 months. All treatment was discontinued after 2 months. Average NAPSI score on each hand was analysed.

Results: A total of 25 participants were recruited, aged 22 to 73 years. Majority were Chinese (72\%), followed by Indian and Malay. There was equal randomisation of treatment to the left and right nail. While there was a rapid significant improvement in average NAPSI score for the control arm at 2 months, the treatment arm had a greater, more sustained improvement of the NAPSI score at 4 months. The average NAPSI score improved for both treatment and control group at 4 months compared to baseline. However, only the NAPSI value improvement in the controls at 2 months compared to baseline was statistically significant $(P=0.0039)$. No severe adverse effects were reported.

Conclusion: To the best of our knowledge, this is the first prospective randomised control trial comparing microneedle technology against conventional topical steroids in nail psoriasis treatment. Our findings demonstrate microneedle technology is as efficacious as topical therapy.
\end{abstract}

Ann Acad Med Singap 2022;51;16-23

Keywords: Microneedle, nail, psoriasis

\section{INTRODUCTION}

Psoriasis is a chronic immune mediated inflammatory skin condition that affects about $2-4 \%$ of the Western populations, with rising incidence over the years. ${ }^{1,2}$ The presentation of psoriasis varies from mild localised plaques to more severe erythrodermic forms, with plaque-type psoriasis being the most common. It frequently affects the skin and scalp, with up to onethird of patients with joint involvement ${ }^{3}$ and over half with nail psoriasis. ${ }^{4}$ Classical psoriatic nail lesions include nail plate pitting, onycholysis, nail bed discolouration, subungual hyperkeratosis and onychodystrophy. Pitting is the most common sign, followed by onycholysis; ${ }^{5}$ but this may not be specific and can be seen in a variety of other nail conditions. The lifetime prevalence of nail involvement for psoriasis patients is estimated to be $80-90 \%{ }^{4}$ and this may present in the absence of cutaneous or joint disease in $5-10 \%$ of patients. ${ }^{6}$ It is likely that the prevalence of nail psoriasis may be underestimated ${ }^{7}$ and commonly overlooked as it is largely asymptomatic in the early stage, especially when patients experience more symptoms from their skin and joint involvement. However, early recognition is important for early intervention as nail psoriasis is considered an indicator for future or early psoriatic joint damage, ${ }^{8}$ and can

\footnotetext{
${ }^{1}$ National Skin Centre, Singapore

${ }^{2}$ Lee Kong Chian School of Medicine, Nanyang Technological University, Singapore

${ }^{3}$ Yong Loo Lin School of Medicine, National University of Singapore, Singapore

* Joint first authors

Correspondence: Dr Hong Liang Tey, National Skin Centre, Dermatology, Singapore 1 Mandalay Road, Singapore 308205.

Email: hltey@nsc.com.sg
} 


\section{CLINICAL IMPACT}

\section{What is New}

- Microneedle patches are as effective as conventional topical steroid treatment at 4 months to serve as additional or alternative treatment for nail psoriasis.

\section{Clinical Implications}

- Self-application of our novel microneedle patches may improve delivery of care and healthcare outcomes for patients compared to intralesional triamcinolone injections that require physician administration and clinic visits.

be associated with longer disease duration, greater disease severity, and greater restrictions in activities of daily living. This can result in lower quality of life as measured with validated instruments such as Dermatology Life Quality Index (DLQI) and Nail Psoriasis Quality of life 10 (NPQ10)..$^{9,10}$

Routine evaluation and early treatment of nail involvement in psoriasis patients is therefore important. Patients should be evaluated in a holistic manner for severity of nail changes, as well as extent of skin and joint involvement. Nail psoriasis is known to be difficult to treat; current treatment modalities include topical therapy, intralesional therapy, photochemotherapy, laser therapy, radiotherapy and systemic therapy, including the use of biologics. Most importantly, patient education on general nail care measures is essential as some patients turn to manicuring practices such as artificial nails and nail extensions to conceal dystrophy, which may in turn irritate nails ${ }^{11}$ and worsen their overall condition.

While topical therapy is a good initial option for patients with proximal nail matrix disease manifesting as pitting of the nails, it has limited efficacy for nail bed disease with subungual hyperkeratosis as topical therapy has suboptimal nail bed access. Beyond topical treatments, procedural therapies such as pulsed dye laser and intralesional corticosteroids have been investigated as possible treatment options. Monthly pulse dye laser therapy has limited efficacy, with long duration of treatment required. ${ }^{12}$ Intralesional injection of corticosteroids can have moderate efficacy but requires in-person weekly injections by a physician for months. ${ }^{13}$ The treatment is very painful, precluding its routine use. Further treatment options such as oral systemic and biologic therapies have been found to be effective but the potential associated adverse side effects limit their use to patients with concurrent more severe cutaneous involvement. ${ }^{14}$ Patients are often fatigued, due to poor efficacy of various treatment modalities and failure to comply to long-term treatments with topical agents. The treatment of nail psoriasis remains challenging due to great difficulty in drug delivery to site of action and possible toxicities of most conventional systemic therapies. With the advent of a new transdermal drug delivery platform in the form of microneedle technology, ${ }_{15}$ the option of a rapid and painless drug delivery to the nail matrix is promising. To investigate the therapeutic effects and advantages of using microneedle technology to deliver topical corticosteroids to psoriatic nails in a rapid and painless way, we conducted a prospective, randomised controlled study to compare microneedle technology with current conventional topical steroid treatment in the treatment of nail psoriasis.

\section{METHODS}

We conducted a 4-month prospective randomised controlled trial in National Skin Centre, a tertiary dermatology centre in Singapore to evaluate treatment response of nail psoriasis following treatment with a self-dissolving microneedle drug delivery patch to administer topical steroids to psoriatic nails. We included all adult patients, aged 21 years and above, with nail psoriasis affecting both hands in varying severity, diagnosed by certified dermatologists. All patients recruited also had classical chronic plaque psoriasis diagnosed by dermatologists. There was consideration of alternative differentials for 2 of the patients. However, their fungal nail microscopy and culture performed were negative, suggesting that the diagnosis of onychomycosis was unlikely. The study excluded those with unilateral nail psoriasis present on one hand had previous localised phototherapy to their nails, oral systemic agents, biologics or have a history of allergy to alcohol swabs, steroids or hyaluronic acid. Patients with recent topical treatment of nails or who were undergoing whole body narrow band ultraviolet B therapy were included but were advised to maintain symmetrical positioning during treatment. All patients underwent a fingernail clipping for microscopy to rule out onychomycosis before enrolment in the study.

This was a left to right intrapatient comparison trial where study participants received sterile microneedle topical steroids treatment on 1 hand and control treatment (topical Daivobet gel containing $50 \mu \mathrm{g} / \mathrm{g}$ calcipotriol and $500 \mu \mathrm{g} / \mathrm{g}$ betamethasone, as dipropionate) on the other hand. Randomisation was performed for each participant to determine treatment allocation for 2 hands. This was done with a computer-generated sequence by a dedicated person who had no further 
involvement in the rest of the study. Patients were taught by research assistants on the way to administer the treatment patch and control treatment.

These dissolving microneedle patches (Fig. 1) were made of sodium hyaluronate as water-soluble matrix material with a total of $0.5 \mathrm{mg}$ triamcinolone embedded in the distal $50 \%$ of the microneedles. The microneedles are $600 \mu \mathrm{m}$ in length and pyramidal in shape. They are prepared via a micromould-based method with a stainless steel master structure consisting of 225 pyramidal needles created using an electrical discharge machining process. The total amount of triamcinolone $(0.5 \mathrm{mg})$ in each microneedle patch to be applied twice weekly was calculated based on the weekly dosage of up to a maximum of $0.1 \mathrm{~mL}$ of $10 \mathrm{mg} / \mathrm{mL}$ injected to proximal nail folds in reported literature. ${ }^{16}$ Alcohol swabs were provided to wipe the nail fold prior to application. The microneedle patches were being held in place over the nail fold area with a nail patch applicator clip (Figs. 2 and 3).

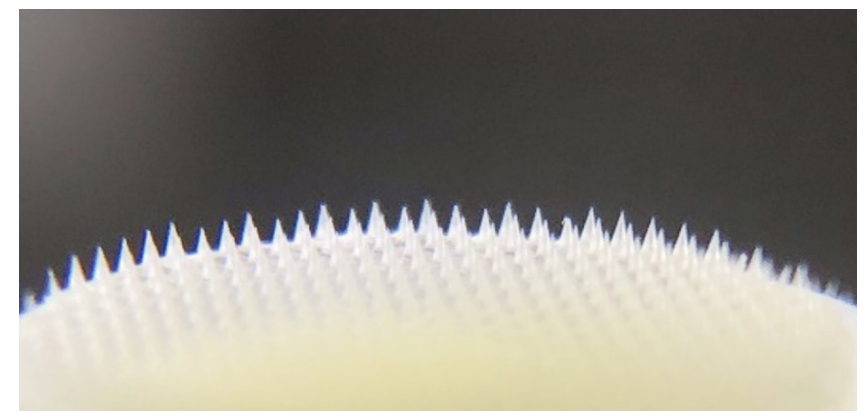

Fig. 1. Microneedle self-dissolving patch.

To assess the severity of psoriatic nail disease, Nail Psoriasis Severity Index (NAPSI) was used in our study. Being one of the most comprehensive assessment tools of psoriatic nail disease used in clinical trials, ${ }^{17}$ it has been validated as a numeric, reproducible, objective and simple tool for evaluation of treatment response of psoriatic nails. In this system, the nail is divided into 4 quadrants and 1 point is awarded if there is any finding of nail matrix and 1 for nail bed change that is seen, per quadrant, or $0-8$ per nail. Nail matrix psoriasis was assessed by the presence of any feature of nail matrix psoriasis, including nail pitting, leukonychia, red spots in the lunula, and crumbling in each quadrant of the nail. Nail bed psoriasis was assessed by the presence of features such as onycholysis, oil drop (salmon patch) dyschromia, splinter haemorrhages, and nail bed hyperkeratosis in each quadrant of the nail. NAPSI scoring was administered by 2 independent

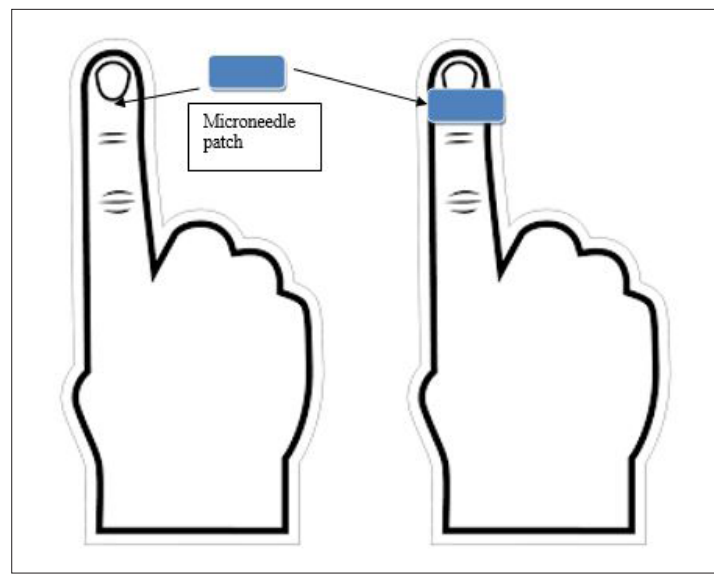

Fig. 2. Application of microneedle patch over horizontal nail fold.

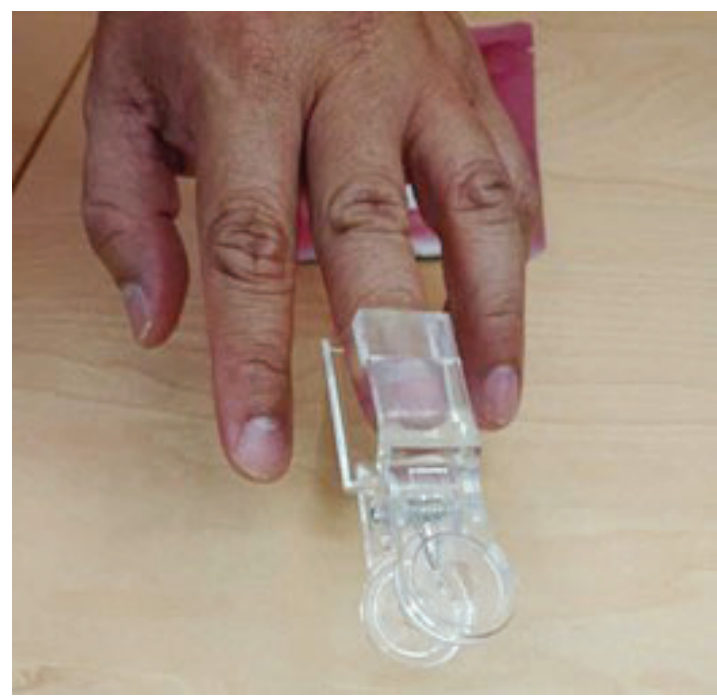

Fig. 3. Clinical photograph of application of nail patch applicator clip.

dermatologists blinded to the treatment assignment at baseline and follow-up visits (2-month and 4-month visits). The average NAPSI nail score of the affected nails on each hand was analysed. This was calculated from the total NAPSI nail score of psoriasis-affected nails divided by the number of affected nails. Other clinical information such as Psoriasis Area Severity Index (PASI), Dermatology Life Quality Index (DLQI) and NPQ10 were measured at baseline and follow-up visits. All recruited patients were reviewed at baseline and at 2 and 4 months after treatment initiation. All treatment was discontinued after 2 months. The study concluded after the third visit (4 months) and all patient information was collated. Any localised side effects to the fingers were also recorded during the follow-up visits. 
In previous studies, ${ }^{18,19}$ patients recruited at baseline from a population with psoriatic nails had a mean NAPSI nail score of about 6 with a standard deviation of 2. Based on the assumption that patients recruited for our study will have similar baseline scores for both the case and control groups, we expect a $1-1.5$ point score difference in nail changes between active and control groups after 4 months of treatment at the end of the study. Based on these assumptions, a sample size of 16-34 is required to detect $1-1.5$ point score difference in nail changes between the 2 groups with statistical power of $80 \%$, at $5 \%$ significance level. Hence, a sample size of 20 patients was decided to achieve the above statistical power at 5\% significance level. The primary outcome of this study was NAPSI scores, and secondary outcomes were PASI, DLQI and NPQ10 scores, all measured at the 2-month and 4-month follow-up visits. Differences between the active against control group, as well as differences between study visits within each treatment group were reported in medians with ranges and means with standard deviations (SD). Wilcoxon signed-rank test was used to compare differences. Statistical significance was assessed at a level of 0.05 . All statistical analysis were performed using $\mathrm{R}$ version 3.5.3 software. The above study design and methods were approved the Singapore institute ethics board prior to initiation.

\section{RESULTS}

A total of 25 patients were recruited for the study, and their demographic characteristics were shown in Table 1. They were aged 22 to 73 years with most of them being males $(76.0 \%)$. Majority of the patients were Chinese (72\%), followed by Indians (3\%) and Malays (3\%). There was approximately equal randomisation of treatment to the left (48\%) and right nail (52\%). Four study participants did not complete the third study visit as they were either lost to follow-up or could not complete their follow-up due to COVID-19 visit restrictions.

There was a rapid significant improvement in average NAPSI score for the control arm at 2 months, while the treatment arm with microneedles had a more sustained and greater improvement of the NAPSI score at 4 months (Fig. 4). The average NAPSI score improved for both the treatment and the control arm in the third visit compared to the first visit, but only the improvement of the NAPSI value in the control group at 2 months compared to baseline was statistically significant $(P=0.004)$ (Table 2). The control group showed higher improvement at 2 months compared with the treatment group but the improvements at 4 months for both groups
Table 1. Demographics of study participants

\begin{tabular}{|c|c|}
\hline Characteristics & $\mathrm{n}=\mathbf{2 5}$ \\
\hline \multicolumn{2}{|l|}{ Age, years } \\
\hline Median (range) & $43(22-73)$ \\
\hline Mean (SD) & $43.28(14.44)$ \\
\hline \multicolumn{2}{|l|}{ Sex, no. (\%) } \\
\hline Male & $19(76.0 \%)$ \\
\hline Female & $6(24.0)$ \\
\hline \multicolumn{2}{|l|}{ Race, no. (\%) } \\
\hline Chinese & $18(72.0)$ \\
\hline Malay & $3(12.0)$ \\
\hline Indian & $3(12.0)$ \\
\hline Others & $1(4.0)$ \\
\hline \multicolumn{2}{|l|}{ Randomisation, no. (\%) } \\
\hline Treatment: Left nail & $12(48.0)$ \\
\hline Treatment: Right nail & $13(52.0)$ \\
\hline
\end{tabular}

SD: standard deviation

were similar (Table 3). These findings reflect that treatment with microneedle patches are at least as effective as the conventional topical steroid treatment. In addition, more than half of patients $(52 \%)$ indicated a score of at least 7 when asked to rate their willingness to use the microneedle patch if it was available in the market on a scale of 0 to 10 . All patients were monitored for adverse events (AE) with only 1 patient with missing $\mathrm{AE}$ data. Of the 9 (37.5\%) patients who reported AE, 7 reported pain, 1 reported discomfort with the clip, 1 reported peeling skin and 1 reported numbness. Of the 7 patients who reported pain, none reported a pain score exceeding 2 out of 10 . The Koebner phenomenon was not observed at the nail folds where microneedles were applied. The overall psoriasis severity remained similar throughout the 4-month study period with similar PASI scores for all 3 visits. However, it was noted that there was a significant decrease in DLQI scores from baseline to 2 months as the study participants received localised therapies targeted to their nails. (Table 4).

\section{DISCUSSION}

Nail psoriasis is prevalent in both psoriasis and psoriatic arthropathy. There is recent increased emphasis on treatment as nail psoriasis is increasingly recognised to have negative impacts on the quality of life and ability 


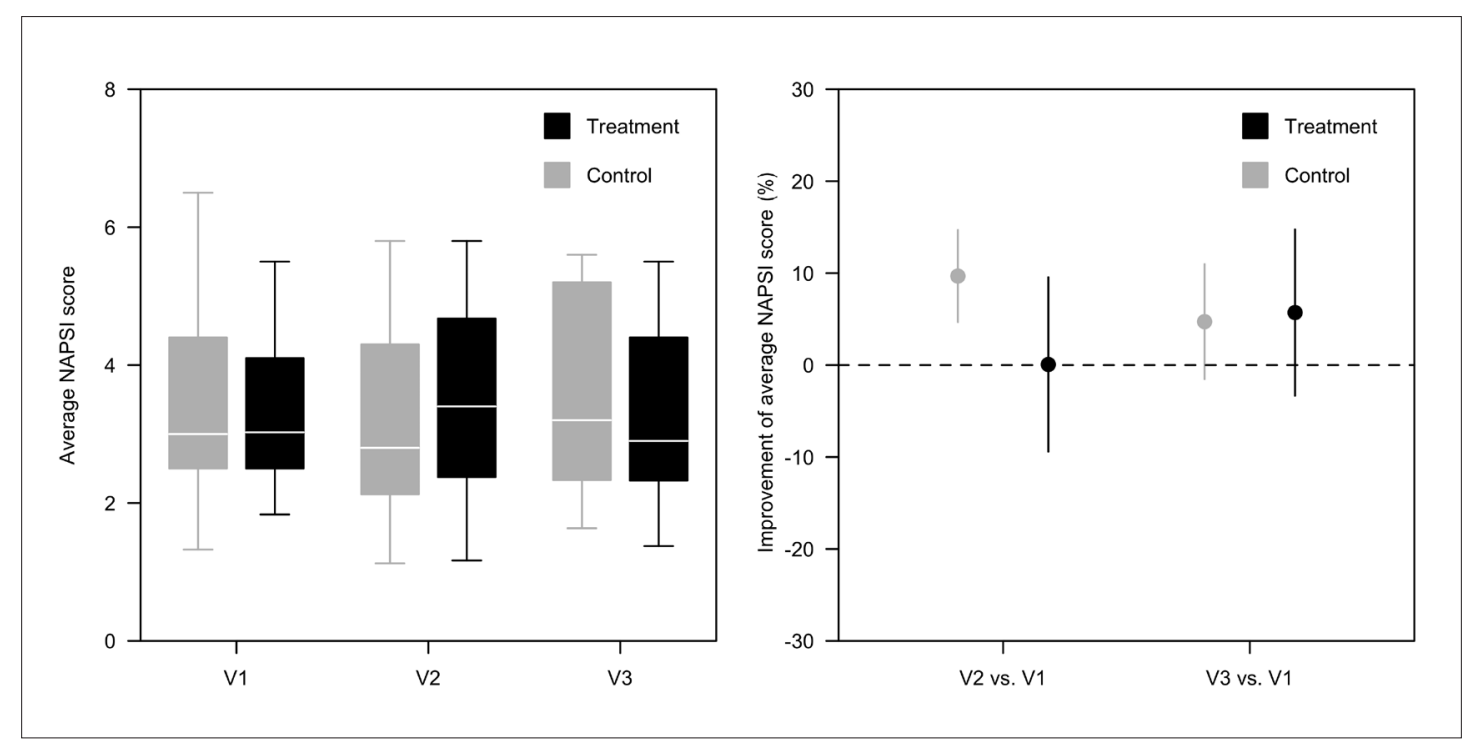

Fig. 4. Comparison of average Nail Psoriasis Severity Index (NAPSI) scores between treatment and control arms across baseline (V1) and follow-up visits (V2 and V3).

$\mathrm{V}$ : visit

to perform daily activities. With limitations to current therapy methods due to poor penetration, ${ }^{20}$ tedious administration and side effects, microneedle technology for drug delivery has come into focus. This is especially relevant in psoriasis, where keratinocyte hyperproliferation causes the formation of prominent plaques that further hinders transdermal delivery. Microneedles can potentially serve as a method of drug delivery considered superior to conventional transdermal delivery as it is known to be minimally invasive, painless, convenient and promotes improved patient compliance. ${ }^{21}$ So far, the use of microneedles has been tested with the administration of methotrexate, ${ }^{22,23}$ cyclosporin ${ }^{24,25}$ and biologics ${ }^{26,27}$ such as anti-TNF-alpha antibody, with good results and minimal side effects. The use of a hyaluronic acid-based microneedle patch had previously been examined in a pilot open trial to treat psoriatic plaques with promising results, but evidence on microneedle patches to treat psoriatic nails is lacking. ${ }^{28}$

Our findings demonstrate that treatment with microneedle patches were at least as effective as conventional topical steroid treatment. The conventional topical steroids treatment had demonstrated a rapid better treatment response compared to the microneedle treatment. While the observed higher NAPSI score at visit 2 for treatment arm was not statistically significant (Table 2), the significantly better improvement scores at visit 2 for control group compared to treatment group could be attributed to a possible delay in any treatment effects from the microneedle compared to the control treatment. Nevertheless, any visible treatment effects seem to be similar between the 2 treatment arms after 4 months, suggesting that the microneedle treatment might have a slower onset or the effects could

Table 2. Average NAPSI score within each group across baseline (V1) and follow-up visits (V2 and V3)

\begin{tabular}{|c|c|c|c|c|c|}
\hline & V1 (n=5) & V2 $(n=5)$ & V3 (n=21) & $\begin{array}{c}P \text { value } \\
(\mathrm{V} 2 \text { vs V1) }\end{array}$ & $\begin{array}{c}P \text { value } \\
(\text { V3 vs V1) }\end{array}$ \\
\hline \multicolumn{6}{|l|}{ Treatment } \\
\hline Median (range) & $3.02(1.83-5.5)$ & $3.4(1.17-5.8)$ & $2.9(1.38-5.5)$ & 0.90 & 0.06 \\
\hline Mean (SD) & $3.42(1.19)$ & $3.42(1.39)$ & $3.31(1.39)$ & & \\
\hline \multicolumn{6}{|l|}{ Control } \\
\hline Median (range) & $3(1.32-6.5)$ & $2.8(1.12-5.8)$ & $3.2(1.63-5.6)$ & 0.004 & 0.10 \\
\hline Mean (SD) & $3.54(1.45)$ & $3.22(1.41)$ & $3.53(1.39)$ & & \\
\hline
\end{tabular}

NAPSI: Nail Psoriasis Severity Index; SD: standard deviation; V: visit 
Table 3. Percentage improvement of NAPSI scores at follow-up visits (V2 and V3) from baseline (V1)

\begin{tabular}{lccc}
\hline & $\begin{array}{c}\text { V1 (n=25) } \\
\mathbf{\%}\end{array}$ & $\begin{array}{c}\text { V2 (n=25) } \\
\mathbf{\%}\end{array}$ & $\begin{array}{c}\text { V3 (n=21) } \\
\mathbf{\%}\end{array}$ \\
\hline Treatment & & & $6.38(-58.18-41.32)$ \\
\hline Median (range) & $3.02(1.83-5.5)$ & $-2.5(-37.5-50)$ & $21 ; 5.71(21.26)$ \\
\hline Mean (SD) & $3.42(1.19)$ & $0.07(24.34)$ & $3.45(-28-36.8)$ \\
\hline Control & & & $21 ; 4.73(14.72)$ \\
\hline Median (range) & $3(1.32-6.5)$ & $9.3(-16.67-37.5)$ & 0.84 \\
\hline Mean (SD) & $3.54(1.45)$ & $9.68(12.84)$ & 0.02 \\
\hline$P$ value & 0.13 & & 0.02 \\
\hline
\end{tabular}

NAPSI: Nail Psoriasis Severity Index; SD: standard deviation

Table 4. Quality of life markers: PASI, DLQI, NPQ10 across baseline (V1) and follow-up visits (V2 and V3)

\begin{tabular}{|c|c|c|c|c|c|}
\hline & V1 & V2 & V3 & $\begin{array}{c}P \text { value } \\
\text { (V2 vs V1) }\end{array}$ & $\begin{array}{c}P \text { value } \\
\text { (V3 vs V1) }\end{array}$ \\
\hline PASI & $\mathrm{n}=24$ & $\mathrm{n}=23$ & $\mathrm{n}=20$ & & \\
\hline Median (range) & $1.65(0-20.1)$ & $1(0-10.7)$ & $1.9(0-17.4)$ & 0.26 & 0.58 \\
\hline Mean (SD) & $2.86(4.60)$ & $2.23(3.02)$ & $2.92(4.18)$ & & \\
\hline DLQI & $\mathrm{n}=25$ & $\mathrm{n}=24$ & $\mathrm{n}=20$ & & \\
\hline Median (rrange) & $4(0-24)$ & $2(0-19)$ & $3.5(0-27)$ & 0.02 & 0.33 \\
\hline Mean (SD) & $6.16(5.75)$ & $4.25(5.02)$ & $6.10(7.52)$ & & \\
\hline NPQ10 & $\mathrm{n}=25$ & $\mathrm{n}=24$ & $\mathrm{n}=19$ & & \\
\hline Median (range) & $0(0-14)$ & $0(0-14)$ & $0(0-15)$ & 0.86 & 0.89 \\
\hline Mean (SD) & $1.40(2.96)$ & $1.54(2.98)$ & $1.84(3.58)$ & & \\
\hline
\end{tabular}

DLQI: Dermatology Life Quality Index; NPQ10: Nail Psoriasis Quality of life 10; PASI: Psoriasis Area Severity Index; SD: standard deviation

possibly be more persistent.

Various matrix materials have been utilised in previous studies to examine the efficacy of drug delivery. Our study used hyaluronic acid as the matrix material for the microneedles in view of its excellent biological properties of biodegradability and non-immunogenicity. The self-dissolvable hyaluronic acid microneedle patch loaded with corticosteroids provides enhanced drug delivery to the nails. These microneedle patches have micron-scale needles that can permeabilise the stratum corneum by creating microchannels in the skin, thereby allowing the embedded corticosteroids to penetrate the nail matrix and bed. These microneedles are long enough to pierce through the barrier but short enough to avoid causing pain. We observed that the treatment arm with microneedles had a more sustained and greater improvement of the NAPSI score at 4 months, but this was not statistically significant. This might be related to the need for a larger sample size or a longer duration of follow-up for the clinical trial.

A strength of this study is its intrapatient design where each patient served as his own case and control for treatment response, which reduces the possible confounders to treatment response. In addition, patients with recent prior treatment with systemic effects were excluded. Our study demonstrates that microneedle patches have the potential to serve as an important adjunct treatment option for nail psoriasis, an alternative to topical therapy, and even intralesional triamcinolone injections. With the potential for self-application of microneedle patches at home, patients have the convenience of home therapy. This will be ideal for patients with busy schedules who are not able to make regular clinic visits for triamcinolone injections. There 
will also be potential time and cost savings as patients need not make multiple clinic visits. Delivery of care to patients and their healthcare outcomes are also likely to improve. Most patients reported no adverse outcomes from applying the nail microneedles and most adverse effects were limited to during the application process. In addition, better designed or customised nail fold applicators could be considered for improved fitting and less discomfort for application.

One of the main limitations of this study is the lack of long-term follow-up, as it is possible that nail dystrophy can relapse just like psoriasis. Furthermore, given the natural speed of nails growth, there might be a lag in any visible nail improvement. Since all treatment was concluded in 2 months but followed up for 4 months, this limited duration might have limited the efficacy of each treatment. Conversely, possible longer-term side effects of microneedle use not observed in this study include infection, irritant contact dermatitis, allergic contact dermatitis, post-inflammatory hyperpigmentation, abnormal scarring, and irritant and allergic granulomas..$^{29}$ A longer duration of treatment and follow-up could show more promising results with the microneedle patch. Further studies with a larger sample size could be performed by expanding on this pilot trial to detect smaller treatment differences with adequate statistical power.

\section{CONCLUSION}

To the best of our knowledge, this is the first prospective randomised control trial comparing the efficacy of microneedle technology against current conventional topical steroid treatment in the treatment of nail psoriasis. While new biologic therapies effective for both plaque psoriasis and psoriatic arthritis are promising for the treatment of nail psoriasis, topical treatments should still be the first-line therapy especially in individuals who have predominantly nail psoriasis with limited systemic involvement. Further research with larger sample sizes is required to further support the use of microneedles to fill treatment gaps in terms of optimal dose adjustments and application frequency, as part of clinical management of nail psoriasis and for further development in this drug delivery field.

\section{Disclosure}

This study was supported by the Ministry of Health's National Medical Research Council, Singapore, under the New Investigator Award (NMRC/CNIG17may-028). HL Tey is supported by the Clinician Scientist Awards (NMRC/CSA-INV/0023/2017 and CSAINV20nov-0003) from the National Medical Research Council, Singapore.

\section{REFERENCES}

1. Kurd SK, Gelfand JM. The prevalence of previously diagnosed and undiagnosed psoriasis in US adults: results from NHANES 2003-2004 (Erratum in J Am Acad Dermatol 2009;61:507). J Am Acad Dermatol 2009;60:218-24

2. Gelfand JM, Weinstein R, Porter SB, et al. Prevalence and treatment of psoriasis in the United Kingdom: a population-based study. Arch Dermatol 2005;141:1537-41.

3. Gladman DD, Antoni C, Mease P, et al. Psoriatic arthritis: epidemiology, clinical features, course, and outcome. Ann Rheum Dis 2005;64(Supp1 2):ii14-7.

4. Jiaravuthisan MM, Sasseville D, Vender RB, et al. Psoriasis of the nail: anatomy, pathology, clinical presentation, and a review of the literature on therapy. J Am Acad Dermatol 2007;57:1-27.

5. Tham SN, Lim JJ, Tay SH, et al. Clinical observations on nail changes in psoriasis. Ann Acad Med Singap 1988;17482-5.

6. Salomon J, Szepietowski JC, Proniewicz A. Psoriatic nails: a prospective clinical study. J Cutan Med Surg 2003;7:317-21.

7. Klaassen KM, van de Kerkhof PC, Pasch MC. Nail psoriasis: a questionnaire-based survey. Br J Dermatol 2013;169:314-9.

8. Langenbruch A, Radtke MA, Krensel M, et al. Nail involvement as a predictor of concomitant psoriatic arthritis in patients with psoriasis. Br J Dermatol 2014;171:1123-8.

9. de Jong EM, Seegers BA, Gulinck MK, et al. Psoriasis of the nails associated with disability in a large number of patients: results of a recent interview with 1,728 patients. Dermatology 1996;193:300-3.

10. Klaassen KM, van de Kerkhof PC, Pasch MC. Nail Psoriasis, the unknown burden of disease. J Eur Acad Dermatol Venereol 2014;28:1690-5.

11. de Berker D. Management of nail psoriasis. Clin Exp Dermatol 2000;25:357-62.

12. Oram Y, Karincaoğlu Y, Koyuncu E, et al. Pulsed dye laser in the treatment of nail psoriasis. Dermatol Surg 2010;36:377-81.

13. Reich K. Approach to managing patients with nail psoriasis. J Eur Acad Dermatol Venereol 2009;23(Suppl 1):15-21.

14. Armstrong AW, Tuong W, Love TJ, et al. Treatments for nail psoriasis: a systematic review by the GRAPPA Nail Psoriasis Work Group. J Rheumatol 2014;41:2306-14.

15. Zhu Z, Luo H, Lu W, et al. Rapidly dissolvable microneedle patches for transdermal delivery of exenatide. Pharm Res 2014; 31:3348-60.

16. Cassell S, Kavanaugh AF. Therapies for psoriatic nail disease. A systematic review. J Rheumatol 2006;33:1452-6.

17. Rich P, Scher RK. Nail Psoriasis Severity Index: a useful tool for evaluation of nail psoriasis. J Am Acad Dermatol 2003;49:206-12.

18. Igarashi A, Kato T, Kato M, et al. Japanese Ustekinumab Study Group. Efficacy and safety of ustekinumab in Japanese patients with moderate-to-severe plaque-type psoriasis: long-term results from a phase 2/3 clinical trial. J Dermatol 2012;39:242-52.

19. Rich P, Bourcier M, Sofen H, et al. Ustekinumab improves nail disease in patients with moderate-to-severe psoriasis: results from PHOENIX 1. Br J Dermatol 2014;170:398-407.

20. Marwah H, Garg T, Goyal AK, et al. Permeation enhancer strategies in transdermal drug delivery. Drug Deliv 2016;23:564-78.

21. Zhao Z, Chen Y, Shi Y. Microneedles: a potential strategy in transdermal delivery and application in the management of psoriasis. RSC Advances 2020;10:14040-9. 
22. Nguyen HX, Banga AK. Delivery of Methotrexate and Characterization of Skin Treated by Fabricated PLGA Microneedles and Fractional Ablative Laser. Pharm Res 2018;35:68.

23. Du H, Liu P, Zhu J, et al. Hyaluronic Acid-Based Dissolving Microneedle Patch Loaded with Methotrexate for Improved Treatment of Psoriasis. ACS Appl Mater Interfaces 2019;11:43588-98.

24. So JW, Park HH, Lee SS, et al. Effect of microneedle on the pharmacokinetics of ketoprofen from its transdermal formulations. Drug Deliv 2009;16:52-6.

25. Jeong HR, Kim JY, Kim SN, et al. Local dermal delivery of cyclosporin A, a hydrophobic and high molecular weight drug, using dissolving microneedles. Eur J Pharm Biopharm 2018;127:237-43.
26. Korkmaz E, Friedrich EE, Ramadan $\mathrm{MH}$, et al. Therapeutic intradermal delivery of tumor necrosis factor-alpha antibodies using tip-loaded dissolvable microneedle arrays. Acta Biomater 2015;24:96-105.

27. Korkmaz E, Friedrich EE, Ramadan $\mathrm{MH}$, et al. Tip-Loaded Dissolvable Microneedle Arrays Effectively Deliver PolymerConjugated Antibody Inhibitors of Tumor-Necrosis-Factor-Alpha Into Human Skin. J Pharm Sci 2016;105:3453-7.

28. Lee JH, Jung YS, Kim GM, et al. A hyaluronic acid-based microneedle patch to treat psoriatic plaques: a pilot open trial. $\mathrm{Br} \mathrm{J}$ Dermatol 2018;178:e24-5.

29. Cary JH, Li BS, Maibach HI. Dermatotoxicology of microneedles (MNs) in man. Biomed Microdevices 2019;21:66 